\title{
VERIFICAÇÃO DE CRITÉRIOS DE DESEMPENHO EM PROJETOS DE ARQUITETURA COM A MODELAGEM BIM
}

\section{CHECKING PERFORMANCE CRITERIA IN ARCHITECTURAL DESIGN WITH BIM}

\author{
Mauro Augusto Silva Junior 1 \\ Instituto de Pesquisas Tecnológicas do Estado de São Paulo, São Paulo, Brasil, email: mauroasjr@gmail.com \\ Cláudio Vicente Mitidieri Filho ${ }^{2}$ \\ Instituto de Pesquisas Tecnológicas do Estado de São Paulo, São Paulo, Brasil, email: claumit@ipt.br
}

\begin{abstract}
Resumo
O presente trabalho tem como objetivo propor uma forma de incorporar a verificação de critérios de desempenho em projetos de arquitetura de edificações habitacionais utilizando a Modelagem de Informação da Construção (Building Information Modeling BIM), como uma alternativa ao método de verificação automática de regras por programas de verificação de modelos BIM. Foi adotado o método baseado na pesquisa construtiva (constructive research) para o desenvolvimento do trabalho no Programa de Mestrado Profissional em Habitação do Instituto de Pesquisas Tecnológicas, IPT. Requisitos e critérios de desempenho constantes da ABNT NBR 15575:2013 Edificações Habitacionais - Desempenho, foram aplicados ao modelo BIM, no desenvolvimento de projetos de arquitetura, de forma que o projetista possa fazer uma verificação ao longo do desenvolvimento do projeto. O resultado foi promissor, considerando a possibilidade de identificar e detalhar aproximadamente um terço dos critérios constantes da ABNT NBR 15575:2013, usando somente a ferramenta da modelagem. Foram identificadas vantagens em utilizar recursos de softwares de modelagem, como etiquetas e tabelas para aplicações de regras e requisitos, que podem ser facilmente constatadas pelo modelo. Desta forma, nota-se que esse novo processo poderá resultar melhores projetos de arquitetura, com informações sobre desempenho mais completas e confiáveis. Se as ferramentas de simulação forem adotadas e associadas a projetos de arquitetura, poderão ser feitas verificações de outros requisitos e critérios, como por exemplo, simulação do desempenho térmico.
\end{abstract}

Palavras-chave: Projeto de arquitetura. Desempenho de edifícios. ABNT NBR 15575. BIM. Verificação de código.

\begin{abstract}
The purpose of this paper is to propose a way to incorporate the verification of performance criteria into architectural designs using Building Information Modeling (BIM), as an alternative to the automatic code validation method by BIM model verification programs. We adopted the Constructive Research method for the development of this study in the Professional Master's Program in Housing of the Institute for Technological Research of the State of São Paulo. Requirements and Performance Criteria in Brazilian Standard ABNT NBR 15575:2013 "Edificações Habitacionais - Desempenho," were implemented as filters on the BIM model so that the designer can make a verification throughout the development of the design. The result was promising considering the possibility of identifying and detailing approximately one-third of the criteria in ABNT NBR 15575:2013, using only the modeling tool. Advantages have been identified in using modeling software features, such as tags and schedules for rules and requirements applications, which can be readily ascertained by the model. In this way, it is noted that this new process may result in better designs, with more complete and reliable performance information. If simulation tools are adopted and associated with architectural designs, other requirements and criteria checks may be made, such as simulation of thermal performance..
\end{abstract}

Keywords: Architectural design. Performance of building. ABNT NBR 15575. BIM. Code checking.

How to cite this article:

SILVA JUNIOR, Mauro Augusto; MITIDIERI FILHO, Claudio Vicente. Verificação de critérios de desempenho em projetos de arquitetura com a modelagem BIM. PARC Pesquisa em Arquitetura e Construção, Campinas, SP, v. 9, n. 4, p. 334-343, dez. 2018. ISSN 1980-6809. Disponível em: <https://periodicos.sbu.unicamp.br/ojs/index.php/parc/article/view/8650453>. Acesso em: 16 out. 2018.

DOl:https://doi.org/10.20396/parc.v9i4.8650453. 


\section{Introdução}

A sociedade passa por uma fase de transformações econômicas, culturais, de valorização de recursos naturais e de adoção constante de novas tecnologias, que vai influenciar os costumes e a vida futura da humanidade. Novas tecnologias também são aplicadas a projetos de edificações, por vários motivos, dentre elas, aquelas que permitem desenvolver projetos de forma mais rápida e também incorporar mais qualidade, com detalhes mais adequados, especificações mais precisas e parâmetros mais consistentes (MOREIRA, 2008).

A atual evolução dos princípios tecnológicos na construção civil está aliada a um novo conceito, o BIM (Building Information Modeling ou Modelagem de Informação da Construção). O BIM procura incluir informações necessárias (custos, prazos, especificações, dados dos fabricantes, parâmetros de desempenho, entre outros) para todo o ciclo de vida da edificação, desde a concepção até a demolição da edificação (incluindo a reutilização), passando pela execução, operação e manutenção (NIBS, 2007).

Outro assunto que ganhou muito destaque no Brasil, principalmente a partir de julho de 2013, é o desempenho de edificações habitacionais, considerando parâmetros que estabelecem exigências de habitabilidade, segurança e durabilidade para habitações. Com a publicação da ABNT NBR 15575:2013 Edificações habitacionais Desempenho (ABNT, 2013 a-f), um importante e indispensável marco para a modernização tecnológica da construção brasileira e melhor desempenho das habitações no país, surgem regras que consideram as exigências dos usuários e dividem responsabilidades entre fabricantes, projetistas, construtores, incorporadores e usuários. A ABNT NBR 15575:2013 contempla cada um dos sistemas que compõem uma edificação habitacional: estrutura, pisos, vedações verticais, coberturas e instalações hidráulicas e sanitárias, além de apresentar requisitos gerais para a edificação como um todo. Só não contempla as fundações e as instalações elétricas e eletrônicas.

Avaliar o desempenho dos sistemas construtivos é um avanço para o setor e constitui o caminho para a evolução de todos que compõem a cadeia da construção civil (CBIC, 2013). Com a ABNT NBR 15575:2013 busca-se a melhora das relações entre os elos da cadeia produtiva, a diminuição das incertezas a partir de critérios objetivos, a instrumentação do Código de Defesa do Consumidor, o estímulo à isonomia competitiva e um instrumento de diferenciação entre as empresas ou entre empreendimentos.

O desenvolvimento de projetos arquitetônicos com especificações técnicas mais precisas influencia ainda mais a relação de consumo e a qualidade da edificação; para isso, os projetistas dispõem de um número significativo de informações técnicas sobre produtos que deverá ser incorporado às atividades de projeto. Para facilitar essa associação de informações, a tecnologia BIM prevê a integração e a atualização de informações durante o ciclo de desenvolvimento de projetos arquitetônicos.

A análise do desempenho de uma edificação ainda é um desafio que precisa ser superado. A utilização de modelos tridimensionais e bibliotecas com informações sobre as características dos materiais, componentes, elementos e sistemas construtivos, será a base para auxiliar a verificação do desempenho com a utilização de ferramentas BIM. Este artigo aborda o uso do BIM no desenvolvimento de projetos de arquitetura aplicando conceitos de desempenho. O tema desempenho é limitado a tópicos que podem ser verificados durante a modelagem BIM e controlados no processo de desenvolvimento de projetos de arquitetura. $\mathrm{O}$ foco principal são projetos de arquitetura de edificações habitacionais. Naturalmente, os mesmos princípios aqui citados podem ser aplicados em projetos de arquitetura para outras finalidades (não habitacional), porém com a devida adequação dos critérios de desempenho para o uso desejado.

Este trabalho tem como objetivo incorporar os conceitos de desempenho ao desenvolvimento de projetos de arquitetura utilizando recursos da modelagem BIM. Como objetivos específicos, buscou-se aplicar os requisitos da ABNT NBR 15575:2013 aos recursos de modelagem, com a incorporação de parâmetros de desempenho.

\section{Desempenho de edificações habitacionais}

Desde a década de 1960, nos países europeus, a definição de desempenho de edificações está associada ao seu comportamento quando em utilização. O edifício é um produto que deve apresentar determinadas características que o capacitem a cumprir objetivos e funções para os quais foi projetado, quando submetido a determinadas condições de exposição e uso.

A construção civil vem sendo impulsionada pelas inovações das tecnologias construtivas demandando novos materiais, técnicas e sistemas construtivos. Neste sentido, a construção vem se tornando cada vez mais industrializada com sistemas construtivos que garantem maior agilidade na execução das edificações. Desta necessidade de industrialização da construção civil surgiu a consciência da avaliação de desempenho e do controle da qualidade na produção dos edifícios habitacionais (MITIDIERI FILHO, 1998).

O conceito de desempenho na construção civil está relacionado ao atendimento das exigências e necessidades dos usuários ao longo do tempo, ou seja, está associado à exigência de durabilidade, e a conceitos como vida útil de 
projeto (VUP) e prazos de garantia, segundo Oliveira e Mitidieri (2012). Considerando os critérios de desempenho é possível estimar o desempenho de uma edificação a partir de uma combinação de métodos para avaliar o produto, prevendo seu comportamento provável ou potencial, quando em utilização normal (MITIDIERI FILHO, 1998). Portanto, a avaliação do desempenho permite verificar se o edifício e suas partes atendem às condições ou aos critérios previamente estabelecidos.

A ABNT NBR 15575 trata-se de uma norma técnica abrangente, que compreende o edifício e suas partes, evidenciando que o atendimento das necessidades dos usuários deve ser vislumbrado desde as primeiras fases de concepção da edificação, em particular desde a fase de projeto. Há um reforço do papel do projeto e consequentemente do projetista, tendo o projeto de arquitetura assumido uma grande importância na definição ou previsão do desempenho da edificação.

A ABNT NBR 15575 "Edificações Habitacionais Desempenho" entrou em vigor em julho de 2013 e contempla seis partes:

- $\quad$ NBR 15575-1 - Requisitos gerais (ABNT, 2013a);

- $\quad$ NBR 15575-2 - Requisitos para os sistemas estruturais (ABNT, 2013b);

- $\quad$ NBR 15575-3 - Requisitos para os sistemas de pisos (ABNT, 2013c);

- $\quad$ NBR 15575-4 - Requisitos para os sistemas de vedações verticais internas e externas (ABNT, 2013d);

- NBR 15575-5 - Requisitos para os sistemas de coberturas (ABNT, 2013e);

- $\quad$ NBR 15575-6 - Requisitos para os sistemas hidro sanitários (ABNT, 2013f);

Cada parte da norma foi organizada por elemento da construção, percorrendo uma sequência de exigências relativas à:

a) Segurança (desempenho estrutural, segurança contra incêndio, segurança no uso e ocupação);

b) Habitabilidade (estanqueidade, desempenho térmico e acústico, desempenho lumínico, saúde, higiene e qualidade do ar, funcionalidade e acessibilidade, conforto tátil); e

c) Sustentabilidade (durabilidade, manutenibilidade e adequação ambiental).

A primeira parte da norma NBR 15575-1 (ABNT, 2013a); Edificações habitacionais possui um caráter de orientação geral, como um índice de referência, remetendo, sempre que possível, às partes específicas (estrutura, pisos, vedações verticais, coberturas e sistemas hidrossanitários). Ela também traz aspectos de natureza geral e critérios que envolvem a edificação como um todo. Apresenta o conceito de vida útil de projeto, a definição de responsabilidades e os conceitos de desempenho em níveis: mínimo (compulsório), intermediário e superior.

Todas as partes da norma contemplam as diversas exigências dos usuários, considerando-se ações de ordem externa à edificação e esforços originados na própria ocupação da edificação. Além disto, são estabelecidos os métodos de avaliação e os níveis de desempenho para cada sistema ou elemento considerado.

Os requisitos especificam os níveis mínimos de desempenho que devem ser alcançados ao longo da vida útil dos sistemas. Os critérios de desempenho expressam a quantificação dos requisitos, definidos com base nas exigências dos usuários, em razão de condições de exposição previamente identificadas, sob as quais está submetida à edificação. Os métodos de avaliação especificam a forma de avaliação de desempenho de um determinado subsistema e podem ser estabelecidos através de análises visuais e de análise de projeto, de ensaios in loco, cálculos, verificações, simulações ou ensaios em laboratório.

A norma estabelece incumbências para incorporadores, construtores, fabricantes, projetistas e usuários para que se atinja e se mantenha o desempenho pretendido durante o prazo de vida útil de projeto. Ao projetista cabe definir os materiais, os produtos e os processos construtivos que serão utilizados, em razão do desempenho requerido, estabelecendo a Vida Útil de Projeto (VUP), que indica o período de tempo em que os requisitos mínimos de desempenho (indicados pela norma) devem ser atendidos pela edificação, supondo a correta manutenção de cada sistema que compõe a edificação. Isto reforça em muito a importância da realização de verificações de desempenho na fase de projeto, de forma a apontar evidências do atendimento de critérios presentes na ABNT NBR 15575.

\section{Verificação de regras}

Um dos diversos usos do BIM é a verificação de regras ou Code Validation definido por CIC (2011) como um processo no qual um programa de validação de código é utilizado para verificar os parâmetros do modelo contra códigos específicos do projeto. Eastman et al. (2009) acrescenta que a verificação automatizada de regras não modifica um projeto, mas o avalia com base na configuração de objetos, suas relações ou atributos. O uso do BIM na verificação de regras incluem coordenação, previsão e validação. É nesse uso do BIM que os dados geralmente são obtidos, do que foi reunido ou gerado, e colocados de forma que possam ser usados para a tomada de decisões (KREIDER; MESSNER, 2013). 
Ainda segundo Eastman et al. (2009) à medida que as ferramentas de verificação forem se aperfeiçoando para desenvolver uma maior conformidade de códigos, esse processo deverá se tornar mais presente no ciclo de desenvolvimento de projetos. Os procedimentos automatizados de verificação de código são um disseminador para a adoção do BIM e para o desenvolvimento de melhores práticas do BIM, consequentemente, contribuindo com informações mais confiáveis e decisões de projetos com maior assertividade.

O processo de verificação de regras passa pelas seguintes etapas (EASTMAN et al., 2009):

1) a interpretação de regras e estruturação lógica de regras para sua aplicação;

2) a preparação do modelo de construção, onde são preparadas as informações necessárias para a verificação;

3) a fase de execução da regra, que realiza a verificação;

4) relatório de resultados de verificação.

Os métodos de implementação de regras podem ser incorporados de diferentes maneiras, entre elas a regra por linguagem de computador, que utiliza a parametrização e ramificação para regras codificadas em linguagem computacional e requer alto nível de experiência para interpretar, escrever e manter seu funcionamento; e tabelas paramétricas, onde os parâmetros e outras classificações podem ser escritos para definir uma classe de regras, instanciados por uma tabela de parâmetros que facilitam a definição de regras para diferentes contextos, e que podem ser aplicadas por usuários sem o conhecimento de programação de computadores, em geral são limitadas pela variedade de condições que podem filtrar e organizar informações. As tabelas paramétricas são um passo intermediário entre facilidade de uso e a generalidade e poder da programação computacional para definir e implementar qualquer regra relevante.

As regras de desempenho de uma edificação exigem analises e simulações derivadas da geometria, localização, materiais empregados e cargas assumidas a edificação que resultam da análise de pressupostos de modelagem para determinar a adequação das regras.

Um exemplo bem-sucedido de verificação de regras, o projeto Construction and Real Estate Network (CORENET) de Cingapura, utiliza o BIM para várias formas de análise, especialmente Building Performance Analysis (BPA), permitindo processos de análise mais precisos e eficientes através do Singapore BIM Guide Version 2 (CORENET, 2013).Para alcançar objetivos de design sustentáveis por meio do BPA baseado em BIM, é necessário transferir informações essenciais, como geometria de modelo precisa, para diferentes formatos de arquivo e software. O BPA visa cobrir verificações de código de construção em planos de construção e conformidade de código para serviços de construção. A verificação de planos inclui regras sobre o controle de construção, acesso sem barreiras, código de incêndio, saúde ambiental, agregado familiar, habitação pública e estacionamento de veículos. Outro exemplo é o sistema da Norueguesa Statsbygg, que realiza a verificação de regras de projeto com base em projetos de construção reais usando múltiplas plataformas para avaliar os requisitos de programa espaciais como verificar a acessibilidade do edifício (Eastman et al., 2009).

Para Solirin, Dimyadi e Lee (2018), um sistema de verificação de regras requer uma maneira de definir regras, que pode ser por meio de uma linguagem. A abordagem baseada em linguagem requer a definição de blocos básicos de regras que podem ser usados para montar regras mais complexas e variáveis. Esse tipo de linguagem é conhecida como Domain Specific Language (DSL). Existem dois gêneros de DSL: interna e externa. A DSL interna utiliza um idioma existente como hospedeiro, estendendo-o e adaptando-o para criar um idioma específico. A DSL externa requer seu próprio analisador e uma nova sintaxe.

Desta forma, este estudo considerou os recursos de verificação de parâmetros em objetos numa ferramenta autoral BIM como uma abordagem de DSL interna para verificação por meio de blocos básicos de regras. Assim, aplicativos de modelagem BIM usados na concepção do projeto, poderiam garantir na proposição da solução o atendimento a, por exemplo, códigos de obra, norma de desempenho, acessibilidade, entre outras.

\section{Método}

$\mathrm{O}$ estudo abordado neste artigo segue o método baseado na pesquisa construtiva (constructive research) ou Design Science Research. A pesquisa construtiva foi adotada, pois pretende-se resolver um problema do mundo real, em que a automação de regras para a verificação de projetos possibilita o avanço tecnológico para o campo da construção civil. $\mathrm{O}$ método da pesquisa construtiva desenvolveu-se em fases, conforme Lukka (2003): a partir da identificação de um problema prático relevante (fase I), examinando-se o potencial de pesquisa (fase II); com o conhecimento teórico e prático da área (fase III) se propõe uma solução (fase IV), implementa-se, testa-se a solução (fase V) e finalmente pondera-se sobre sua aplicabilidade (fase VI). Este artigo enfatiza a apresentação das fases III a VI.

No exame do potencial da pesquisa foram analisadas as possibilidades de verificação, com recursos de uma ferramenta de modelagem BIM, de parâmetros de desempenho, na fase do projeto de arquitetura, 
considerando-se o Guia AsBEA de boas práticas em BIM (ASBEA, 2015), proposto pela Associação como instrumento prático de trabalho do arquiteto. A intervenção explícita do pesquisador é uma característica da pesquisa construtiva, sendo que a construção desenvolvida e implementada deve ser considerada como um instrumento de teste e uma tentativa para ilustrar, testar ou refinar uma teoria existente ou desenvolver uma nova teoria (LUKKA, 2003). Contou-se desta forma com a experiência profissional nos softwares BIM da Autodesk, principalmente no aplicativo Revit, para aplicar os conceitos de desempenho com recursos da modelagem da informação, e com a experiência na verificação prática de critérios de desempenho. A proposta de verificação dos parâmetros de desempenho foi aplicada, de forma piloto, em um modelo BIM de um projeto de edificação, especificamente no pavimento tipo, destinado a habitação residencial.

Uma vez identificado os recursos da ferramenta autoral BIM com potencial de verificação de parâmetros de desempenho, partiu-se para a proposição da solução, implementação e teste. Finalmente, para ponderar sobre a aplicabilidade da solução, avaliou-se o trabalho realizado levantando-se as facilidades de gestão e análise da informação, as dificuldades encontradas e perspectivas futuras pela adoção de um novo conceito.

\section{Proposição da solução e implementação}

Entre os recursos da ferramenta autoral Autodesk Revit adotados na pesquisa, destacam-se os seguintes recursos que podem ser aplicados para a verificação de modelos:

\section{Etiquetas (TAG)}

A utilização de etiquetas (TAG) apresentando informação constante em parâmetro associado à norma de desempenho, que lê as informações que constam nos parâmetros e aplicam nas vistas (planta) do projeto, como exemplo o item 8.8 Dificultar a propagação do incêndio, da fumaça e preservar a estabilidade estrutural da edificação (8.3 - Partes 3, 5 e 6; 8.4 - Parte 4 da ABNT NBR 15575:2013), onde o projetista utiliza uma biblioteca de componentes que contem parâmetros que indicam o Tempo Requerido de Resistência ao Fogo (TRRF) dos elementos da edificação. As especificações referentes ao TRRF podem ser visualizadas na planta do projeto (TAG) facilitando a tomada de decisões do projetista.

Na Figura 1 o Tempo Requerido de Resistência ao Fogo (TRRF) para o sistema de piso é indicado em sua caixa de propriedades, e a aplicação da informação ao projeto pelo recurso de etiqueta (Tag) do Autodesk Revit, que lê as informações preenchidas no campo do respectivo parâmetro, e transcreve automaticamente para a vista do projeto.

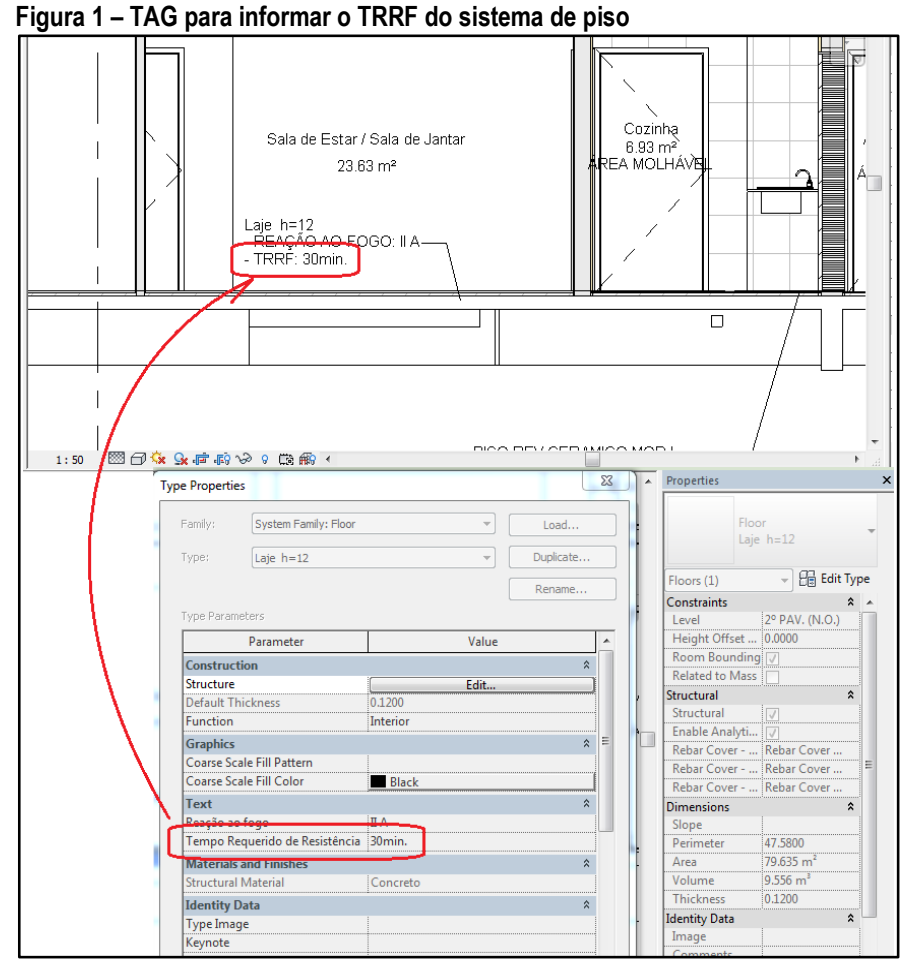

Fonte: Silva Junior (2016, p.73)

\section{Filtros e cores}

A aplicação de filtros e regras de visualização, identificando com coloração não conformidades ou aspectos que precisam ser mais bem especificados conforme a norma técnica foi outro recurso utilizado no desenvolvimento da pesquisa, como, por exemplo, o item 9.3 Segurança na circulação (9.2 e 9.3 - Parte 3) da ABNT NBR 15575:2013. O projetista utiliza a aplicação de regras para verificar os desníveis dos ambientes do projeto e, com o auxílio de filtros, é possível sua visualização na planta, no corte ou na elevação mostrada no projeto (view templates); é possível, então, identificar por cores os pisos com desníveis que precisam de especificações e detalhes típicos (Figura 2).

\section{Tabelas (Schedules)}

Tabelas são recursos disponíveis para aplicação de regras de formatação condicionais, identificando não conformidades ou necessidades de especificação em conformidade com itens da norma. Entre os itens que se utilizaram deste recurso no estudo desenvolvido está o 11.3 Aberturas para ventilação (11.3 - Parte 4) da ABNT NBR 15575:2013; o projetista utiliza uma biblioteca de componentes que contem parâmetros que especificam o atendimento às normas técnicas vigentes. As especificações referentes ao parâmetro informativo de atendimento a aberturas para ventilação podem ser aplicadas e visualizadas nas vistas do projeto, e também através de tabelas que direcionam o item em desacordo (em destaque) para uma vista, facilitando a identificação e tomada de decisões do projetista (Figura 3). 
Figura 2 - Filtros e cores para identificar desníveis de pisos - Segurança na circulação

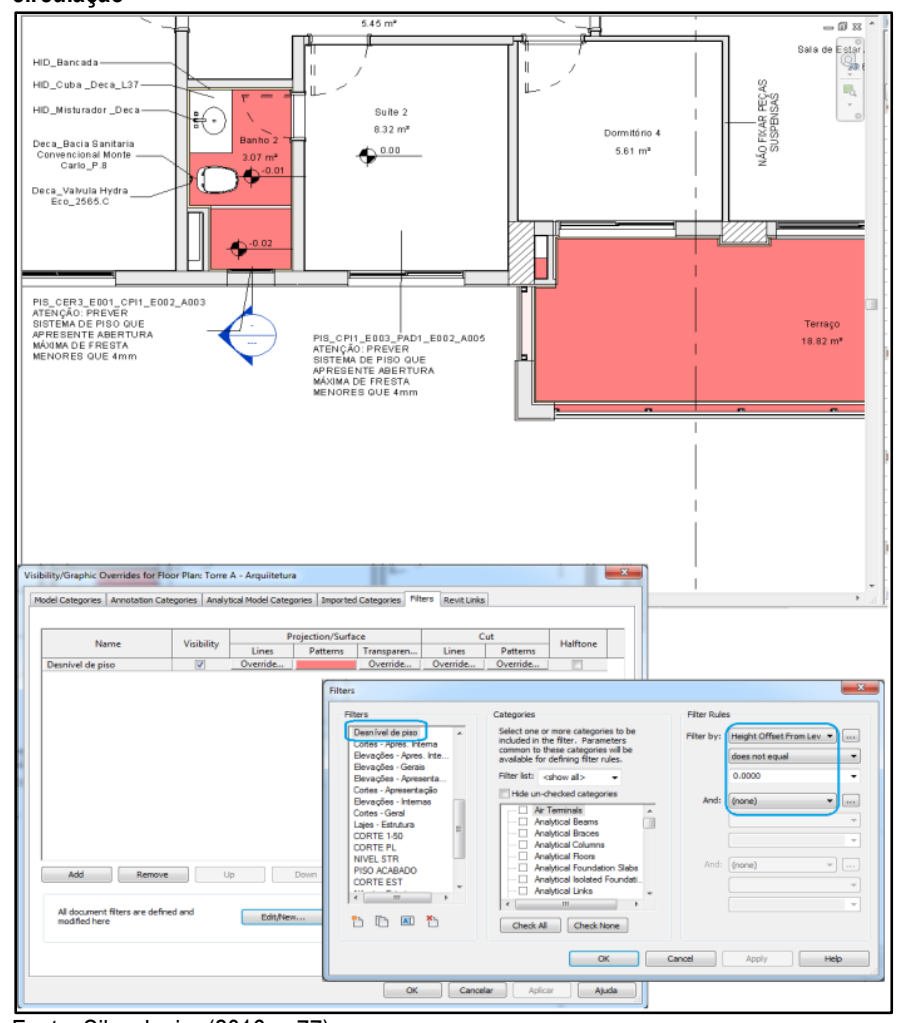

Fonte: Silva Junior (2016, p.77).

Figura 3 - Tabelas identificando aberturas de ventilação

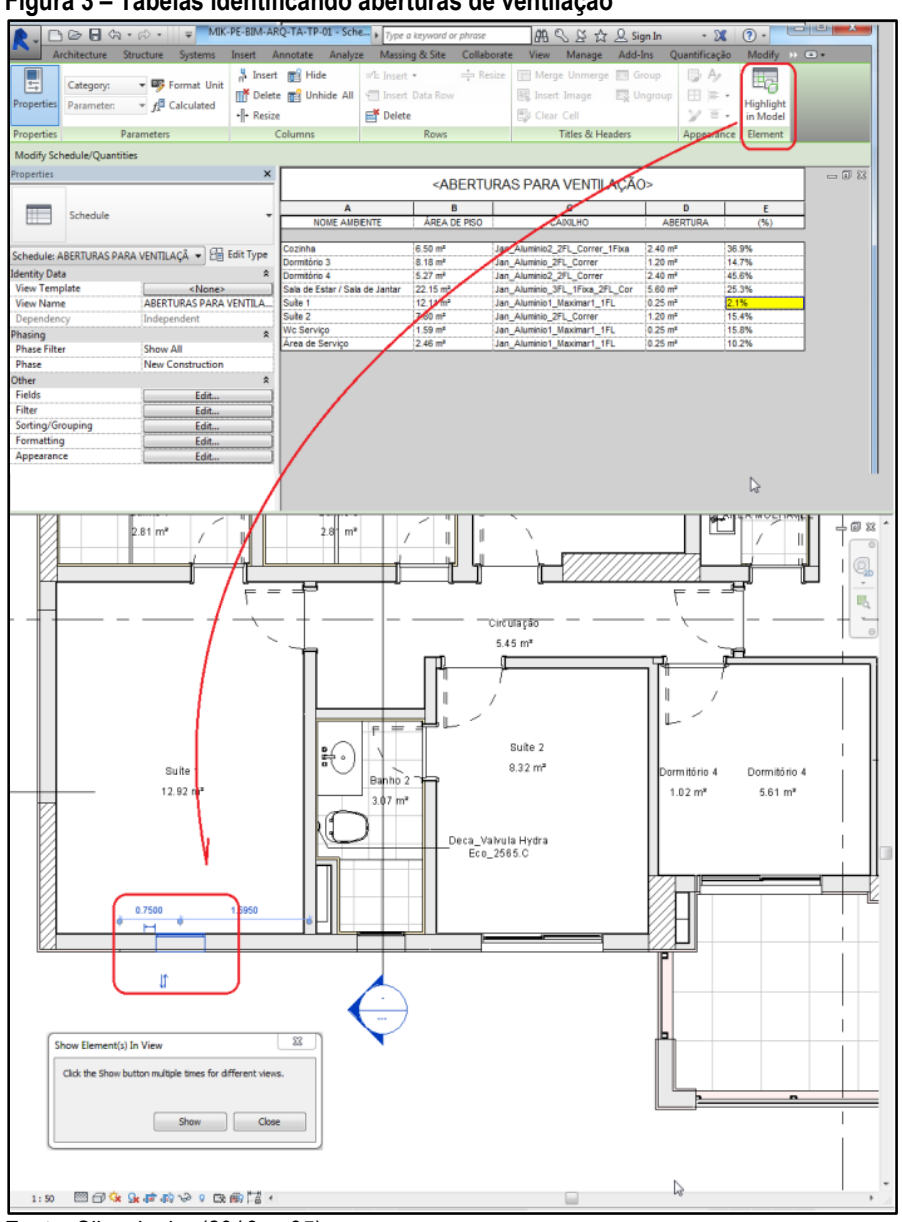

\section{O potencial da solução}

Para cada um dos itens da norma analisou-se as ações propostas no "Guia para arquitetos na aplicação da norma de desempenho" (ASBEA, 2016), contrapondo-se com recursos existentes de verificação e extração de informação do modelo. Identificaram-se os seguintes recursos aplicáveis: etiquetas apresentando informação constante em parâmetro associado à norma de desempenho; filtros e regras de visualização, identificando com coloração não conformidades com itens da norma, ou necessidades de especificação conforme a norma; regras de formatação condicionais, identificando não conformidades ou necessidades de especificação conforme a norma; e tabelas sumarizando itens da norma. A aplicação destes recursos foi demonstrada na seção anterior. O Quadro 1 enumera os itens da NBR 15575-1 (ABNT, 2013a), NBR 15575-2 (ABNT, 2013b), NBR 15575-3 (ABNT, 2013c), NBR 15575-4 (ABNT, 2013d), NBR 15575-5 (ABNT, 2013e) e NBR 15575-6 (ABNT, 2013f) com potencial de verificação a partir do modelo BIM e o tipo de recurso aplicável.

\section{Discussão}

Verificou-se que aproximadamente $30 \%$ dos itens da norma de desempenho são verificáveis por esta proposta de checagem. Entretanto, a distribuição de verificação é variável (Figura 4).

\section{Aplicabilidade da solução}

O estudo mostrou que a incorporação de parâmetros de desempenho ao modelo BIM, utilizado para desenvolvimento de projetos de arquitetura, possibilita a criação de regras que facilitam: a identificação de requisitos e critérios; a identificação de recursos correlatos disponíveis no software, como tabelas e etiquetas (Tag); a gestão e a organização das informações de um projeto, atendendo total ou parcialmente as principais exigências da ABNT NBR 15575:2013. O estudo aplicado considerou a possibilidade de identificar e informar aproximadamente um terço dos critérios constantes da ABNT NBR 15575:2013 com o uso do projeto em BIM. Os demais requisitos e critérios relativos ao desempenho estrutural, à estanqueidade, ao desempenho térmico e acústico, ao desempenho lumínico, à funcionalidade e acessibilidade, ao conforto tátil e antropodinâmico e à adequação ambiental, além dos já identificados pela ferramenta de modelagem, podem ser também considerados no projeto com emprego do BIM, expandindo-se este trabalho, desde que estejam disponíveis dados de desempenho dos elementos e sistemas da construção e que sejam associadas ferramentas de simulação. 
Quadro 1 - Itens da norma de desempenho verificáveis com recursos nativos da ferramenta de autoria Autodesk Revit (com base no Guia AsBEA, 2015)

\begin{tabular}{|c|c|c|}
\hline Item da norma & Tema & $\begin{array}{l}\text { Item da norma e } \\
\text { recurso BIM } \\
\text { aplicável }\end{array}$ \\
\hline 7 & SEGURANÇA ESTRUTURAL & $5 / 8$ \\
\hline 7.4 & 4. Resistência às solicitações de cargas - peças suspensas (7.3.1 - P.2; 7.3.1 - P.3; 7.3.1 - P.4; 7.4.1 - P.5) & TAG \\
\hline 7.9 & 9. Resistência a cargas verticais concentradas em Pisos (7.5.1 - Parte 3) & TAG \\
\hline 7.10 & 10. Resistência a ações transmitidas por portas (7.5.1-Parte 4) & TAG / TABELA \\
\hline 7.14 & 14. Solicitações dinâmicas - válvulas de descarga (7.2.1-Parte 6) & TABELA \\
\hline 7.18 & 18. Ação do granizo e outras cargas acidentais em telhados (7.5.1 - Parte 5) & TAG / TABELA \\
\hline 8 & SEGURANÇA CONTRA FOGO & $1 / 5$ \\
\hline 8.5 & 5. Dificultar a ocorrência de inflamação generalizada (8.2 - Parte3, 4 e 5; 8.2 - Parte 4) & TAG / TABELA \\
\hline 8.8 & 8. Dificultar a propagação do incêndio - preservar a estabilidade estrutural (8.3 - P.3, 5 e $6 ; 8.4$ - P.4) & TAG / TABELA $\left(^{*}\right)$ \\
\hline 9 & SEGURANÇA NA UTILIZAÇÃO & $3 / 7$ \\
\hline 9.2 & 2. Coeficiente de atrito da camada de acabamento (9.1 - Parte 3) & TAG \\
\hline 9.3 & 3. Segurança na circulação (9.2 e 9.3 - Parte 3) & FILTRO $(*)$ \\
\hline 9.6 & $\begin{array}{l}\text { 6. Risco de choques elétricos e queimaduras em sistemas de equipamento de aquecimento e em } \\
\text { eletrodomésticos ou eletroeletrônicos }(9.3 .1 \text { - Parte } 1,9.1 \text { - Parte } 6)\end{array}$ & TAG \\
\hline 9.11 & 11. Manutenção e operação Aterramento de sistemas de coberturas metálicas. (9.2.5 - Parte 5) & TAG \\
\hline 9.13 & 13. Permitir utilização segura aos usuários $(9.3$ - Parte 6$)$ & TAG / TABELA \\
\hline 9.14 & 14. Temperatura de utilização da água (9.4 - Parte 5) & TAG / TABELA \\
\hline 10 & ESTANQUEIDADE & $2 / 3$ \\
\hline 10.1 & $\begin{array}{l}\text { 1. Estanqueidade a fontes de umidade externas à edificação e a animais (10.2-P.1; 10.1-P.3; 10.1-P.4 .10.1-P. } \\
\text { 5) }\end{array}$ & TAG / TABELA \\
\hline 10.2 & $\begin{array}{l}\text { 2. Estanqueidade a fontes de umidade internas à edificação - operação e manutenção do imóvel em condições } \\
\text { normais de uso (10.3 - Parte } 1 ; 10.3 \text { - Parte } 3 ; 10.2 \text { - Parte } 4 ; 10.3 \text { - Parte } 4)\end{array}$ & TAG / TABELA \\
\hline 11 & DESEMPENHO TÉRMICO & $3 / 3$ \\
\hline 11.1 & 1. Adequação de paredes externas (11.2 - Parte 4) & TAG / TABELA \\
\hline 11.2 & 2. Isolação térmica da cobertura (11.2 - Parte 5) & TAG / TABELA \\
\hline 11.3 & 3. Aberturas para ventilação (11.3 - Parte 4) & FILTRO / TABELA (*) \\
\hline 12 & DESEMPENHO ACÚSTICO & $4 / 4$ \\
\hline 12.1 & 1. Isolação acústica de paredes externas (12.2.1 - Parte 1) & TAG / TABELA \\
\hline 12.2 & 2. Isolação acústica entre ambientes (12.3.1 - Parte 1) & TAG / TABELA \\
\hline 12.3 & 3. Níveis de ruído permitidos na habitação (12.3 - Partes 3,4 e 5$)$ & TAG / TABELA \\
\hline 12.4 & 4. Nível de ruído de impacto em coberturas acessíveis de uso coletivo (12.4 - Parte 5) & TAG / TABELA \\
\hline 13 & DESEMPENHO LUMÍNICO & $2 / 2$ \\
\hline 13.1 & 1. lluminação natural (13.2.1 e 13.2.3 - Parte 1) & FILTRO / TABELA \\
\hline 13.2 & 2. lluminação artificial (13.3.1 - Parte 1) & TABELA \\
\hline 14 & DURABILIDADE E MANUTENIBILIDADE & $0 / 8$ \\
\hline 15 & SAÚDE, HIGIENE, QUALIDADE DO AR & $0 / 9$ \\
\hline 16 & FUNCIONALIDADE E ACESSIBILIDADE & $1 / 9$ \\
\hline 16.1 & 1. Altura mínima de Pé-direito (16.1 - Parte 1) & FILTRO / TABELA \\
\hline 17 & CONFORTO TÁTIL E ANTROPODINÂMICO & $2 / 5$ \\
\hline 17.1 & 1. Conforto na operação dos sistemas prediais (17.2 - Parte 6$)$ & TAG / TABELA \\
\hline 17.2 & 2. Conforto tátil e adaptação ergonômica (17.2.1 Parte 1) & TAG / TABELA \\
\hline 18 & ADEQUAÇÃO AMBIENTAL & $1 / 9$ \\
\hline 18.9 & 9. Uso racional da água (18.1 - Parte 6) & TABELA \\
\hline
\end{tabular}

Nota: $\left(^{\star}\right)$ Ver exemplo em PROPOSIÇÃO DA SOLUÇÂO E IMPLEMENTAÇÃO. Fonte: Os autores.

Figura 4 - Distribuição do potencial de implementação da verificação em Autodesk Revit por item da norma

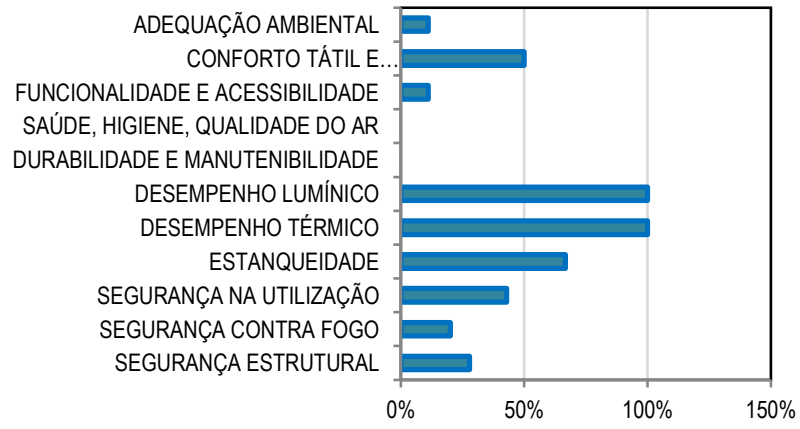

Fonte: Os autores.
Entre os resultados obtidos, a utilização de uma biblioteca de elementos construtivos com informações padronizadas de desempenho para a adequação de paredes externas e coberturas, pode otimizar o trabalho do projetista na transcrição dos dados de transmitância e capacidade térmica no projeto, assim como a identificação das aberturas para ventilação de cada ambiente da edificação. Estes dados, se necessário, podem ser exportados juntamente com as coordenadas geográficas do edifício, enviadas para análise em softwares específicos como o EnergyPlus. 
A utilização dos modelos BIM no desenvolvimento de projetos de arquitetura, juntamente com a aplicação de parâmetros de desempenho da edificação, como uma alternativa ao método bidimensional tradicionalmente utilizado pelos projetistas e arquitetos, é o objetivo deste estudo, que foi atendido e mostrou que existem vantagens como a aplicação automática de etiquetas (Tag) que reconhecem as informações constantes nos parâmetros de cada componente do modelo e podem, em geral, ser visualizadas em plantas e planilhas, facilitando a tomada de decisões do projetista e a correspondente especificação de componentes e elementos, a partir de informações qualificadas.

\section{Conclusão}

Segundo Solirin, Dimyadi e Lee (2018), existe um progresso significativo de sistemas automatizados de verificação de regras baseados em BIM. Entretanto, ainda é requerido transpor o estado de prova de conceito para a implementação no mundo real. Estes autores avaliam que nenhuma abordagem única é capaz de abranger todo o espectro de requisitos para sistemas automatizados de verificação de regras. Neste contexto, a solução aqui proposta é, agregadora, ampliando o conjunto necessário de formas automatizadas de verificação de regra, assim como antecipando o momento de utilização no processo de projeto.

A solução aqui proposta pode também ser adotada para a verificação da qualidade de modelos BIM no atendimento à normas e premissas de projetos apresentadas no escopo de modelagem BIM pelo contratante do projeto.

No Brasil a aplicação da ABNT NBR 15575:2013 é uma boa oportunidade para a melhoria do desempenho das habitações brasileiras, e o BIM vem ao encontro para agregar valor ao projeto, uma vez que o modelo tem uma grande importância no processo de visualização dos elementos e componentes construtivos em três dimensões, facilitando o desenvolvimento do projeto. Além disso, o "I" do Building Information Modeling tem uma relevância maior ainda no resultado do processo, possibilitando a extração de uma informação mais precisa e confiável, principalmente nas alterações de projeto e nas mudanças de fase que requerem mais do projetista, na visualização dos requisitos impostos pelo processo de desenvolvimento de projetos de arquitetura, pelas constantes novas normas e pela garantia e confiabilidade das informações geradas pelo projeto de arquitetura.

A adoção do modelo BIM para desenvolvimento de projetos de arquitetura em conformidade com a ABNT NBR 15575:2013 constitui uma alternativa de investigação futura nos resultados do processo de projeto, viabilizando o desenvolvimento com a utilização das tecnologias disponíveis no mercado, destacando a importância de seus parâmetros e processos a favor dos profissionais do setor da construção civil, com projetos mais qualificados, competitivos e com adequado nível de desempenho para o mercado.

Para estudos e trabalhos futuros são feitas as seguintes sugestões:

- validação da aplicação de parâmetros de desempenho em projetos de arquitetura através de aplicações práticas, no sentido de eventualmente ampliar os itens ou parâmetros considerados;

- análise de ferramentas de simulação BIM para complementação da avaliação na fase de projeto, integrando outros parâmetros de desempenho;

- desenvolvimento de ferramentas computacionais de integração, para automatizar análises de parâmetros de desempenho; e,

- desenvolvimento de relatórios a serem gerados, com evidências da adoção de parâmetros de desempenho na fase de projeto.

\section{Notas}

(1) Uma versão resumida deste artigo foi apresentada e publicada nos Anais do ENCONTRO NACIONAL DE TECNOLOGIA NO AMBIENTE CONSTRUÍDO de 2018.

\section{Referências}

ABNT - ASSOCIAÇÃO BRASILEIRA DE NORMAS TÉCNICAS. NBR-15575-1: edificações habitacionais: desempenho: parte 1: requisitos gerais. Rio de janeiro: 2013a.

ABNT - ASSOCIAÇÃO BRASILEIRA DE NORMAS TÉCNICAS. NBR-15575-1: edificações habitacionais: desempenho: parte 2: requisitos para os sistemas estruturais. Rio de janeiro: $2013 \mathrm{~b}$.

ABNT - ASSOCIAÇÃO BRASILEIRA DE NORMAS TÉCNICAS. NBR-15575-1: edificações habitacionais: desempenho: parte 3: requisitos para os sistemas de pisos. Rio de janeiro: 2013c. 
ABNT - ASSOCIAÇÃO BRASILEIRA DE NORMAS TÉCNICAS. NBR-15575-1: edificações habitacionais: desempenho: parte 4: sistemas de vedações verticais internas e externas - SVVIE. Rio de janeiro: $2013 \mathrm{~d}$.

ABNT - ASSOCIAÇÃO BRASILEIRA DE NORMAS TÉCNICAS. NBR-15575-1: edificações habitacionais: desempenho: parte 5: requisitos para sistemas de coberturas. Rio de janeiro: $2013 \mathrm{e}$.

ABNT - ASSOCIAÇÃO BRASILEIRA DE NORMAS TÉCNICAS. NBR-15575-1: edificações habitacionais: desempenho: parte 6: sistemas hidrossanitários. Rio de janeiro: 2013f.

ABNT - ASSOCIAÇÃO BRASILEIRA DOS ESCRITÓRIOS DE ARQUITETURA. Guia AsBEA boas práticas em BIM Fascículo II. São Paulo, 2015. Disponível em: http://www.asbea.org.br/download/Guia_Bim_AsBEA_Fasciculo_2.pdf. Acesso em: 22 set. 2015.

ASBEA -ASSOCIAÇÃO BRASILEIRA DOS ESCRITÓRIOS DE ARQUITETURA. Guia BIM para arquitetos na aplicação da norma de desempenho ABNT NBR 15.575. São Paulo, 2015. Disponível em: http://www.caubr.gov.br/wpcontent/uploads/2015/09/2 guia_normas_final.pdf. Acesso em: 05 mar. 2016.

CBIC - CAMARA BRASILEIRA DA INDÚSTRIA DA CONSTRUÇÃO. Desempenho de edificações habitacionais: Guia orientativo para atendimento à norma ABNT NBR 15575/2013. Brasília, 2013.

CIC - COMPUTER INTEGRATED CONSTRUCTION RESEARCH PROGRAM, BIM Project Execution Planning Guide Version 2.1. May, The Pennsylvania State University, University Park, PA, USA, 2011.

CORENET - Construction and Real Estate NETwork. Singapore BIM Guide Version 2 ,Singapura, 2013. Disponível em: https://www.corenet.gov.sg/media/586132/Singapore-BIM-Guide_V2.pdf Acesso em: 28 out.2018

EASTMAN, C. et. al. Automatic rule-based checking of building designs. Automation In Construction, [s.1.] v. 18, n. 8, p.10111033, dez.2009 Elsevier BV. DOI:https://doi.org/10.1016/j.autcon.2009.07.002

KREIDER, R. G.; MESSNER, J. I. The uses of BIM: Classifying and Selecting BIM Uses. Version 0.9, September, The Pennsylvania State University, University Park, PA, USA, 2013. Disponível em: http://bim.psu.edu/Uses/the_uses_of_bim.pdf. Acesso em: 28 out.2018

LUKKA, K. The constructive research approach. Case study research in logistics. Publications of the Turku School of Economics and Business Administration, Series B, v. 1, p. 83-101, 2003.

MITIDIERI FILHO, C.V. Avaliação de desempenho de componentes e elementos construtivos inovadores destinados a habitações: proposições específicas à avaliação do desempenho estrutural. São Paulo. 1998. 218f. Tese (Doutorado) Departamento de Engenharia Civil, Escola Politécnica da Universidade de São Paulo. São Paulo, 1998.

MOREIRA, T, P, F, A influência da parametrização dos softwares CAD arquiteturais no processo de projetação arquitetônica. 2008 165f. Dissertação (Mestrado) - Mestrado de arquitetura e urbanismo, área de concentração: tecnologia. Universidade de Brasília. Brasília, 2008

NBIS - NATIONAL INSTITUTE OF BUILDING SCIENCES. NBIMS - National Building Information Modeling Standards Version 1, Part 1: overview, principles and methodologies. Washington: NBIS, 2007. Disponível em: http://www.wbdg.org/pdfs/NBIMSv1_p1.pdf. Acesso em: 01 dez.2013

OLIVEIRA, L.; MITIDIERI FILHO, C. O PROJETO DE EDIFÍCIOS HABITACIONAIS CONSIDERANDO A NORMA BRASILEIRA DE DESEMPENHO: ANÁLISE APLICADA PARA AS VEDAÇÕES VERTICAIS. Gestão \& Tecnologia de Projetos, v. 7, n. 1, p. 90-100, 14 jun. 2012. DOI:https://doi.org/10.4237/gtp.v1i1.208

SILVA JUNIOR, M.A. Parâmetros de desempenho incorporados em projetos de arquitetura com o uso de aplicativo de modelagem BIM. 2016. Dissertação (Mestrado em Habitação: Planejamento e Tecnologia) - Instituto de Pesquisas Tecnológicas do Estado de São Paulo. Área de concentração: Tecnologia em Construção de Edifícios, São Paulo, 2016. Disponível em: https://www.ipt.br/dissertacoes_interna_click_pdf.php?id=894\&link=cassiopea.ipt.br/teses/2016_HAB_Mauro_Augusto.pdf

SOLIHIN, W; DIMYADI, J; LEE, Y-C. In search of open and practical language-driven BIM-based automated rule checking systems. In: MUTIS, I.; HARTMANN, T. (ed.). Advances in Informatics and Computing in Civil and Construction 
Engineering: Proceedings of the 35th CIB W78 2018 Conference: IT in Design, Construction, and Management. Cham: Springer, 2018. p. 577-586.

\author{
${ }^{1}$ Mauro Augusto Silva Junior \\ Arquiteto e urbanista. Mestre em Habitação: Planejamento e Tecnologia da Construção de Edifícios no Instituto de Pesquisas \\ Tecnológicas do Estado de São Paulo (IPT). Endereço postal: Rua Eng. Isaac Garcez, 400 São Bernardo do Campo, São Paulo, \\ Brasil, 09619-110
}

\title{
${ }^{2}$ Claudio Vicente Mitidieri Filho
}

Engenheiro Civil. Doutor em Engenharia Civil pela Universidade de São Paulo. Endereço postal: Av. Prof. Almeida Prado, 532 Cidade Universitária Armando de Salles Oliveira, São Paulo, São Paulo, Brasil, 05508-901 